\title{
Systems biology approach reveals the mystery of aging origin
}

\author{
A. Khalyavkin ${ }^{1,2 *}$, V. Krut'ko ${ }^{2,3}$ \\ ${ }^{1}$ Emanuel Institute of Biochemical Physics RAS, Moscow, Russia \\ ${ }^{2}$ Institute for Systems Analysis FRC CSC RAS, Moscow, Russia \\ ${ }^{3}$ Sechenov First Moscow State Medical University, Moscow, Russia \\ *e-mail:antisenesc@mail.ru
}

Key words: origin of aging, systems approach, self-maintenance, environmental influence

Motivation and Aim: An overview of our current knowledge of mortality statistics and biology of aging can provide better understanding of some general peculiarities of macro-systems in different environments and might lead us to the development of useful approaches to the origin of aging and its control and especially explain why we age despite having potentially ageless somatic stem cells that can even reverse aging.

Methods and Algorithms: We used the critical analysis of the array of published experimental findings and our particular interpretation of these findings in order to unite at the one conception many separate and various data obtained from the molecular level up to the level of population.

Results: We suggested that the control systems of a potentially ageless organism are able to sustain a physiological regimen of complete self-maintenance strictly within a certain range of values and changes in external conditions known as 'environmental pressure'. The bell-shaped and the U-shaped curves characterizing 'dose - effect' relationship are well-known features, which describe the regularities of interaction between biological systems and their environments. For this reason even a potentially non-senescent body must start to age under inadequate condition (like a non-melting piece of ice taken out from the deepfreeze inevitably starts to melt at the temperatures above zero Celsius). This conception is totally consistent with existing patterns of mortality and with agelessness potential of somatic stem cells [1-6].

Conclusion: There is no need to build up and explore too complicated "systems models of intrinsic aging" to understand the origin of this mainly extrinsic root cause of natural aging. For this reason a simple phenomenological black-box approach with Input-Output analysis is ample. Here Input refers to the environmentally dependent initial force of mortality, whereas Output is a rate of age-related increase of mortality force.

\section{References}

1. Khaliavkin A.V. (2001) Influence of environment on the mortality pattern of potentially non-senescent organisms. General approach and comparison with real populations. Advances in Gerontology. 2:46-49.

2. Khalyavkin A.V., Yashin A.I. (2007) Nonpathological senescence arises from unsuitable external influences. Annals of the New York Academy of Sciences. 1119:306-309.

3. Khalyavkin A.V. (2012) From macro- to nano-systems and back in search of the primary cause and control of aging. Proceedings of International Conference "Instabilities and control of Excitable Networks: From Macro- to Nano-Systems", 69-76 (Dolgoprudny). DOI 10.13140/2.1.4408.0326

4. Khalyavkin A.V. (2013) Phenoptosis as genetically determined aging influenced by signals from the environment. Biochemistry (Moscow). 78(9):1001-1005. DOI 10.1134/S0006297913090058

5. Khalyavkin A.V., Krut'ko V.N. (2014) Aging is a simple deprivation syndrome driven by a quasiprogrammed preventable and reversible drift of control system set points due to inappropriate organismenvironment interaction. Biochemistry (Moscow). 79(10):1133-1135.

6. Khalyavkin A.V., Krut'ko V.N. (2018) How regularities of mortality statistics explain why we age despite having potentially ageless somatic stem cells. Biogerontology. 19(1):101-108. 